\section{The effects of instructions, fixed rate of presentation, and complexity on free looking time}

\author{
HENRY WAIBEL and RICHARD W. THOMPSON \\ Western Washington State College, Bellingham, Washington 98225
}

The effect of stimulus complexity, prior experience with a short or long fixed rate of presentation (FRP) and S's knowledge concerning the purpose of the experiment on free looking time (FLT), was examined. Results indicated that under all conditions, Ss view complex stimuli longer than simple. A long FRP produced longer FLT than did a short FRP for Ss uninformed of the purpose of the experiment, but FRP had no effect on FLT for Ss who were informed.

Recently, Leckart, Gehres, and Thornton (1970) showed that the presence or absence of the $\mathrm{E}$ during free looking time (FLT) experiments and the S's information concerning the purpose of the experiment can have dramatic effects of S's FLT. When $\mathrm{E}$ is absent FLT is longer than when $E$ is present, and when $S$ is informed of the purpose of the experiment, FLT is shorter than when $S$ is given typical nonspecific instructions or is told that $E$ is interested in measuring S's GSR to different stimuli.

Results of earlier experiments have demonstrated a number of other variables influencing FLT. For example, FLT has been shown to vary directly with stimulus complexity, FLT being longer for complex stimuli than for simple stimuli (Leckart, 1966). It has also been shown that preexposing $S$ to a series of stimuli presented at fixed short or fixed long durations will result in shorter or longer FLT, respectively, than if $S$ has had no such preexposure (Leckart, Keeling, \& Bakan, 1966). The present experiment was conducted to investigate the effects of instructions as they might interact with other variables that have been shown to affect FLT. Specifically, the present experiment investigated two fixed rates of presentation (FRP 7 and $21 \mathrm{sec}$ ), stimulus complexity (low, medium, and high), and instructions (which either revealed or did not reveal the purpose of the experiment) on FLT.

\section{METHOD}

Subjects

The Ss were 28 niale and female student volunteers from the introductory psychology course at Western Washington State College.

\section{Apparatus}

The stimuli were selected from a set of 59 colored slides of common objects which had been rated previously by 20 student $S$ s on a 7.point complexity scale. A mean complexity rating was computed for each slide, and nine slides at low, middle, and high complexity were selected as stimuli for the experiment. The mean and standard deviation of complexity ratings for the three sets of slides were: low, 1.71 and 0.12 ; middle, 3.86 and 0.04 ; and high, 6.11 and 0.19 . The stimuli were projected by a Kodak Carousel 650 projector which was located $225 \mathrm{~cm}$ from a white lenticular screen. The $S$ sat $180 \mathrm{~cm}$ from the screen on which a $70 \times 95 \mathrm{~cm}$ image was projected. The slides could be controlled either by $S$, by means of a hand switch, or automatically, by a timer located in an adjacent room. A BRS Electronics printout counter located in the adjacent room recorded FLT for each slide when $S$ controlled their presentation.

\section{Procedure}

In the first part of the experiment, $14 \mathrm{Ss}$ viewed a 21-sec presentation of each slide (Group FRP-21) and 14 Ss viewed three $7-5 e c$ presentations of each slide (Group FRP-7). A different random order of slides was used for each presentation. The Ss were told in this part of the experiment that they would view some slides which would be presented automatically, that their memory for the slides would not be tested, and that after they had viewed the slides they would be given further instructions. The $\mathrm{E}$ was not present iis either the first or second part of the experiment.

In the second part of the experiment the Ss viewed the same 27 slides again, S controlling the duration of each slide. Half the Ss in Groups FRP-7 and FRP-21 were told that the duration that $S$ viewed each slide would be automatically recorded for further study (informed group). The other half of the Ss in Groups FRP-7 and FRP-2 I were told they would view each slide again, that they could change the slide when they wished, and that they would be given further instructions after they had viewed all the slides (uninformed group). Again, all Ss were assured they would not be asked to recall anything about the slides.

The experiment was a factorial design with independent factors of FRP ( 7 and $21 \mathrm{sec}$ ), instructions (informed and uninformed), and repeated measures of FLT over the three levels of stimulus complexity (low, medium, and high).

\section{RESULTS AND DISCUSSION}

Mean FLT in seconds was computed for each $S$ for the three levels of complexity, and these data were used to compute an analysis of variance with factors of FRP instructions, and repeated measures over complexity. Mean FLT in seconds for the four groups for each level of complexity are presented in Table 1 . The analysis yielded a significant effect of FRP $(F=10.46, \quad d f=1 / 24, \quad p<.005)$, instructions $(F=9.68, d f=1 / 24$, $\mathrm{p}<.005)$, complexity $(\mathrm{F}=8.66$, df $=2 / 49, p<.001)$, and the interaction of FRP by Instructions $(F=7.70, d f=1 / 24$, $p<.025)$. Subsequent analysis of the interaction indicated a significant effect of FRP for uninformed Ss $(F=11.32$, $\mathrm{df}=1 / 12, p<.01)$ but not for informed Ss $(F<1)$. Further analysis by $t$ tests indicated that uninformed 21-sec FRP Ss had longer FLT than either the uninformed 7 -sec FRP Ss $(t=5.78, d f=40, p<.001)$ or the informed $21 \mathrm{sec}$ FRP $S s(t=4.15$, $\mathrm{df}=40, \mathrm{p}<.001)$.

Consistent with the data of Leckart, Gelires, and Thornton (1970), the results

Table 1

Mean and Standard Deviation of Free Looking Time for All Groups at Three Levels of Complexity

\begin{tabular}{|c|c|c|c|c|c|c|c|c|}
\hline \multirow[b]{3}{*}{ Group } & \multicolumn{8}{|c|}{ Complexity } \\
\hline & \multicolumn{2}{|c|}{ Low } & \multicolumn{2}{|c|}{ Medium } & \multicolumn{2}{|c|}{ High } & \multicolumn{2}{|c|}{ Overall } \\
\hline & $\overrightarrow{\mathrm{X}}$ & $\mathrm{S}$ & $\bar{x}$ & $\mathrm{~S}$ & $\overline{\mathrm{X}}$ & $\mathrm{S}$ & $\ddot{\mathrm{X}}$ & $\mathrm{S}$ \\
\hline \multicolumn{9}{|l|}{ I:RP-7 } \\
\hline Informed & 3.51 & 2.26 & 3.90 & 2.22 & 5.04 & 3.66 & 4.15 & 2.87 \\
\hline Uninformed & 4.36 & 2.14 & 4.62 & 1.75 & 4.95 & 1.88 & 4.65 & 2.04 \\
\hline \multicolumn{9}{|l|}{ I:RP-2I } \\
\hline Informed & 3.73 & 1.75 & 4.78 & 2.15 & 5.97 & 3.18 & 4.83 & 2.60 \\
\hline Uninformed & 12.03 & 6.14 & 13.34 & 6.81 & 15.25 & 6.35 & 13.54 & 6.57 \\
\hline Overall & 5.91 & 5.02 & 6.67 & 5.48 & 7.80 & 5.95 & & \\
\hline
\end{tabular}


of this experiment indicated that the instructions given $S$ greatly influence the duration of FLT, even eliminating the effect of FRP in Ss informed of the purpose of the experiment. However, complexity of the stimulus seems to affect duration of FLT independent of instructions or FRP. These results suggest, consistent with Leckart et al, that in experiments concerned with the effect of various variables on FLT, $S$ should be given instructions that do not reveal the purpose of the experiment. The ruse of wiring $S$ to measure physiological responses, such as the GSR suggested by Leckart et al, would appear to provide an adequate rationale to the $\mathbf{S}$ for the purpose of the experiment and not to interfere with measurement of FLT.

\section{REFERENCES}

LECKART, B. T. Looking time: The effects of stimulus complexity and familiarity.
Perception \& Psychophysics, 1966, 1, 142-144.

LECKART, B. T., GEHRES, L., \& THORNTON, G. Looking time: Experimenter and instruction effects. Perception \& Psychophysics, 1970, 8, 54-56.

LECKART, B. T., KEELING, K. R., \& BAKAN, P. The effect of rate of presentation on free looking time. Perception \& Psychophysics, 1966, 1, 107-109.

(Accepted for publication October 18, 1970.) 